\title{
Inszenierung als Beruf. Der Fall Guttenberg
}

\author{
Viktor Winkler
}

Rezension von Oliver Lepsius / Reinhart Meyer-Kalkus (Hg.) (2011). Inszenierung als Beruf. Der Fall Guttenberg. Suhrkamp, Berlin.

Es handelt sich um das erste Buch zur Affäre Guttenberg. Die Biographie von Eckart Lohse und Markus Wehner (Guttenberg. Biographie, München 2011) war bereits vor den Plagiatsvorwürfen vom Februar 2011 geschrieben worden und konnte den Sturz des Porträtierten nur im aktualisierten Vorwort berücksichtigen. Während der vorübergehend Entthronte meint, es in Amerika besser zu haben, beweist der von Oliver Lepsius und Reinhart Meyer-Kalkus herausgegebene, auf einen Workshop am Berliner Wissenschaftskolleg zurückgehende Band, dass der Rücktritt Guttenbergs mehr bereithält als nur Lehren über den richtigen Umgang mit öffentlichen Vorwürfen.

Das Buch ist ein großer Gewinn. Es handelt sich weder um eine Nacherzählung der Ereignisse vom Februar 2011 noch, wie erste Rezensionen angenommen haben, um eine Abrechnung mit dem Plagiator. Auch der Versuchung, durch Übersetzung in unverständliche akademische Kategorien das nachzuerzählen, was Jedem bekannt war, ist der Band nicht erlegen. Statt dessen geht es in dreizehn ebenso sachkundig wie fesselnd geschrieben Beiträgen sowie einer analytisch brillianten Einordnung von Oliver Lepsius um das Exemplarische am Sturz Guttenbergs, um den Fall also und nicht nur um das Fallen. Journalisten, Literaturwissenschaftler, Historiker, Soziologen, Kunsthistoriker, Medienwissenschaftler und Philosophen widmen sich in einer "interdisziplinären Fallstudie" (Oliver Lepius) jenen Aspekten, an denen wie in einem Brennglas sehr Grundsätzliches, auch grundsätzlich Neues abzulesen ist: Zur neuen Rolle der sozialen Netzwerke (Bernhard Pörksen / Hanne Detel), der Mediendemokratie (Uwe Pörksen), dem Verhältnis von politischem und Wissenschaftssystem (Gustav Seibt, Thomas Steinfeld, Petra Gehring) oder der politischen Konstitution Deutschlands im Jahr 2011 (Johannes von Müller, Tilman Allert, Nils Minkmar).

Besonders reizvoll ist, wie der Band sich die Mühe macht, zentrale Texte des Gescheiterten Stück für Stück zu analysieren, vom schwülstigen und unfreiwillig komischen, leider nicht abgeschriebenen Vorwort der Doktorarbeit, über die mit atemberaubender Chuzpe Schuldeingeständnis und Schuldvorwurf, Abwehr und Angriff, Wahrheit und Lüge verbindenden Verteidigungssätze im Februar 2011, bis hin zur von Selbstmitleid durchzogenen Rücktrittsrede vom 1. März 2011. Es geht dabei um mehr als um übliche Politikersprache und ihre Kritik oder Karikierung. Vor allem Heinrich Deterings und Sebastian Diziols brilliante Analysen der grammatisch verstolperten, aber sehr aufschlussreichen Rücktrittser- klärung des Ministers (tote Soldaten, Verantwortung, „Anstand”, „Herzblut”, „Schmerzen”, „Charakter”, „Kraftlosigkeit”) machen nachdenklich. Nicht so sehr die Unverschämtheit, mit der Guttenberg statt dem Plagiat den Schutz „seiner" Soldaten vor den Medien zum Rücktrittsgrund macht, ist bemerkenswert, sondern vielmehr die Antiquiertheit von Stil, Begriffen und Wendungen. Es ist das pudrige Pathos der politischen Sprache des 19., nicht des 20. Jahrhunderts, wohl auch eines militärisch geprägten Adels, vielleicht aber nur der dürftig angeeignete Stil eines jungen Dorfadligen mit humanistischer Erziehung, der täglich durch die schlosseigene Ahnengalerie laufen musste und meinte, so müssten sie reden, die historischen Größen in großen historischen Momenten.

Dieser radikal antimodernistische, mal zackig-autoritäre, mal martialische, mal operettenhafte Stil prägte die Sprache Guttenbergs von Beginn an, wie der Band nachdrücklich belegt. Es ist Populismus, aber es ist ein Populismus mit untypischem Inhalt: Steif statt kumpelhaft, elitär statt proletarisch, angepasst statt aufrührerisch. Dass er dafür von großen Teilen der Öffentlichkeit geliebt statt verspottet wurde und wird, mag vielleicht wie wenig Anderes den Charakter einer angepassten Generation verdeutlichen: anständig, „Werte”-verliebt, häuslich, adrett, höflich. Immerhin: Die Doktorarbeit wurde zum Skandal, die Rücktrittsrede nicht. Vielleicht hätte das in dem Band noch mehr betont werden können. In der devoten Huldigung des pomadigen Adligen aktualisiert sich (hoffentlich) nicht so sehr antidemokratisches Ressentiment, in ihr macht sich wohl eher eine Mittelschichtjugend Luft, die sich seit Langem von „1968”, aber auch der Kultur der 90er Jahre verabschieden will. Für sie sind Mimik, Gang, Kleidung, Frisur des Ministers nicht albern, sondern schick.

Der Band ist kein theoretisches Traktat, sondern, sehr praktisch, ein Füllhorn an Denkanstössen. Sie führen weg von den überschätzen rhetorischen Talenten des Lokalpolitikers aus Kulmbach hin zu den viel bedeutenderen kollektiven Sehnsüchten, die auch ein Anderer hätte bedienen können. Tatsächlich manifestierte sich im Fall Guttenberg wie selten zuvor die Sehnsucht der Bürger (nicht notwendig der Wähler) nach einem Ende der Parteiendemokratie, wie sie - historisch durchaus nicht zwingend - von Adenauer einst persönlich mit grosser Energie etabliert worden ist. Guttenberg selbst tat alles, um diese Aversionen unablässig zu füttern, auch und gerade noch in seiner Rücktrittserklärung, in der er sich schamlos zum Märtyrer des Politik- und Medienbetriebes erklärte. Aber der meist blasiert-verkrampfte, früh als mässiger Staatsschauspieler entlarvte Guttenberg war, dies zeigt eben nicht nur sein desaströses Krisenmanagement, nicht der Meister der neuen Möglichkeiten. Das kann und das wird man 
besser machen. Gleichwohl handelte mit Guttenberg, der alle Popularitätsumfragen, aber noch nie eine Wahl gewonnen hat, zum ersten Mal ein neuer Typus im Sinn Max Webers: Ein Politiker, der sich nahezu vollständig auf medial vermittelte (und erzeugte) Zustimmung stützt und nicht auf jene seiner Parteigremien (Schröder war insoweit nur ein Anfänger). Vielleicht ist das die wichtigste der zahlreichen Veränderungen, die der sehr gelungene Band nachvollziehbar macht.

\title{
Vorerst gescheitert
}

\author{
Johannes Staemmler
}

Rezension von Karl-Theodor zu Guttenberg und Giovanni di Lorenzo (2011). Vorerst gescheitert. Herder.

„Lieber inkompetent als schuldig“ titelte Der Spiegel über Robert Murdoch, der versuchte, sich aus der Abhör-Affäre seiner Boulevard-Blätter in England herauszuwinden. Der Titel passt aber nicht weniger gut auf ein Buch, das einige qua seiner Existenz für ein Ärgernis halten. In Vorerst gescheitert reden der ehemalige Verteidigungsminister zu Guttenberg und der ZEIT-Chefredakteur di Lorenzo über alles, was $2011 \mathrm{im}$ Leben des heutigen Privatiers zu Guttenberg passiert ist, wie es dazu kam und was daraus wird.

Der einst beliebteste Politiker Deutschlands trat am 3. März 2011 von seinen Ämtern mit der Begründung zurück, er halte dem öffentlichen Druck nicht mehr stand und fühle sich am Ende seiner Kräfte. Die Debatte drehe sich, so der Minister, nur noch um Fehler von ihm aus der Vergangenheit und nicht um die Soldaten, die in Afghanistan kämpften und auch stürben. Zu Guttenberg verließ das Land.

Das Buch beginnt gleich mit den Fragen, die damals offen blieben. Haben sie betrogen, Herr Guttenberg? Und damit beginnt ein Reigen verwundener Fabulierungen, in denen der zu Guttenberg den Vorwurf des Vorsatzes von sich weist. Er begründet die Vielzahl nicht zitierter fremder Texte (ergo Plagiate) mit der ebenso unfassbaren Anzahl an Datenträgern, die ihn während der Arbeit begleiteten und über die er den Überblick verlor. Diese sich über viele Jahre hinziehende Arbeit sei eine „Dummheit“ gewesen, deren Abschluss er selbst auf ein gewisses Maß an „Hochmut“ zurückführt. Hätte er betrügen wollen, dann hätte er es cleverer gemacht. Dem Leser bleibt die Wahl zwischen Regen und Traufe auf der Suche nach einer schlüssigen Erklärung.

Bevor der Frage nachgegangen wird, ob der Wissenschaftler zu Guttenberg und der derzeit außer Dienst stehende Politiker zur selben Persönlichkeit gehören, sei noch eine Bemerkung in eigener Sache erlaubt. Als die Debatte um die Doktorarbeit des Ministers abzuflauen drohte, formulierten Doktoranden in Berlin einen Offenen Brief an die Kanzlerin mit der Bitte, sich in dieser Sache genauer zu erklären. Sie fürchteten darum, dass nicht nur alle mühsame wissenschaftliche Kleinarbeit zur Makulatur werden könnte, sondern dass der gesamte Wissenschaftsstandort Deutschland sich als FeelGood-Thema ohne Substanz entpuppt. Innerhalb kürzester Zeit versammelten sich 60.000 Menschen mit ihren Unterschriften unter diesem Brief als Signal ihrer Empörung.
Auf Seite 47 lässt der Privatier nun ungewollt einen Einblick auf sein Wesen zu. Es hätte ihm tatsächlich erst sehr spät gedämmert, dass sich irgendwer durch sein Handeln gekränkt gefühlt haben könnte. Schließlich müsse man aber bedenken, dass er seine Arbeit unter anderen Ausgangsbedingungen geschrieben habe. Das Ringen um Mitleid nimmt seinen Lauf.

Diese Haltung steht beispielhaft für weite Teile des Buches, in dem der Satz „Ich würde es wieder genauso machen.“ der häufigste ist. Wir begegnen einem Süchtigen, dem die Drogen Aufmerksamkeit und Gehorsam entzogen wurden. Herr zu Guttenberg fordert - er bittet nicht - Fairness in der Bewertung seiner Handlungen. Er begründet rational, warum er auf dem Broadway oder als Kampfjetpilot posierte. Auch spricht er von sich als prinzipienfest und mutig, im Stillen auch nachdenklich und vor allem den Menschen zugewandt und bereit, ihnen beim Tragen ihrer Bürden zu helfen. Er redet über sich als einen, dem das ungefragt Helfen in die Wiege gelegt wurde und der gar nicht anders kann, als im Rampenlicht zu stehen, zum Besten der anderen.

In der Verbindung seines eigenen Wohls mit dem Wohl der Bürger gelingt es ihm, Mitgefühl beim Leser zu erzeugen. Man erwischt sich dabei, ihm glauben zu wollen, dass er das Beste wollte. Und genau darin liegt die Gefahr zumindest des Buches. Giovanni di Lorenzo schafft es nicht, das Erlösungsversprechen offensichtlich $\mathrm{zu}$ machen. Er versucht ihn $\mathrm{zu}$ bedrängen, erliegt ihm aber am Ende selbst. Der Leser wünscht sich schließlich, dass zu Guttenberg zurückkommt, denn dann geht es dem Freiherrn besser und dem Leser auch. Auch lässt er sein politisches Programm durchschimmern, wenn er explizit das Unbehagen mit „Führung“ für beendet erklärt und stattdessen heraushebt, dass von Deutschland Führung erwartet würde. „Wir müssen es nur geschickt und klug machen (...) “ (S. 165).

Es steht nach der Lektüre des Buches außer Zweifel, dass Herr zu Guttenberg die politische Bühne wieder betreten wollen wird. Er braucht sie und lebt von ihrer Aufmerksamkeit. Nur lässt es seine Persönlichkeit nicht zu, sich in Demut über sein Handeln zu üben. Das Buch legt schillernd aber klar offen, auf was wir uns dann einstellen müssen. Es ist eine Lektüre, die, wenn man sie einrahmt mit Werken Webers, Freuds und Shakespeares, sich zum unfertigen Skript einer düsteren Heldensage verdichtet. Bleibt anzukündigen, dass wir Wissenschaftler alles tun werden, um ihre Vollendung zu verhindern. 